\title{
Patient expectations and regulatory needs in the risk-based drug development of cystic fibrosis
}

\author{
EDINA PALLAGI*, ILDIKÓ CSÓKA \\ Institute of Pharmaceutical Technology and Regulatory Affairs, University of Szeged, \\ Eötvös Str. 6, 6720 Szeged, Hungary \\ Corresponding author: Edina Pallagi \\ Email:pallagi.edina@szte.hu
}

Received: 27 November 2020 / Accepted: 16 December 2020

\begin{abstract}
Aims: Cystic Fibrosis (CF) is a rare, chronic, progressive, multi-organ disease. It needs life-long, complex therapy with many difficulties for patients to follow. New therapy development also has difficulties owing to regulatory and economic challenges.

Methods: This study evaluates the intervention possibilities of therapy management in CF, taking into consideration the aspects of patient adherence and Quality of Life (QoL), regulatory and economic backgrounds. It presents a Quality by Design (QbD)-based knowledge and Risk Assessment (RA)-based evaluation method.

Results: The RA-based evaluation showed that the most highly critical intervention points in the CF therapy-management with greatest positive estimated impact are: increasing of the social support, increasing of the level of patient education and improvement of the potentials of therapy monitoring. The degree of adherence in CF depends mainly on the degree of progression and the complexity of the therapy. New drug combination and new medicine development were found as they have lower critical effect of the patient's QoL. Conclusion: The risk-based exploration of the therapy interventions can help in finding the factors which have the highest effect on the therapy success and patient adherence. They can be the alternative of a time and cost consuming new medicinal research and development.
\end{abstract}

Keywords: Cystic fibrosis, CF therapy management, Quality of Life, Risk assessment, risk-based therapy management

\section{Introduction}

\subsection{Cystic fibrosis and Quality of Life}

Cystic fibrosis (CF) is an autosomal recessive genetic disorder. $\mathrm{CF}$ disease is caused by mutations in the gene encoding for the cystic fibrosis transmembrane conductance regulator (CFTR). Chemically, CTFR is a protein that acts as a chloride channel in the body [1]. The genetic mutation of CTFR in CF disease results in the defect of chloride and sodium transport, which is manifested in viscous secretions of different exocrine tissues [2]. The damaged, non-regularly acting CTFR forms in the plasma membrane channels and makes it permeable to chloride, bicarbonate, and other anions as well [3]. Due to this transmembrane ionic transport defect, such microenvironment is created which favours the colonization of bacteria that eventually become resistant to antibiotics. [3] In $\mathrm{CF}$ disease the following organs and tissues are mainly affected: the respiratory tract (lung, etc.), the pancreas, the gastrointestinal tract, sweat glands and other exocrine tissues. The increased viscosity makes secretions difficult to clear, which results in chronic respiratory disease as well as other pathologies including pancreatic insufficiency, obstructive hepatic and biliary abnormalities, distal intestinal obstruction syndrome, and reduced fertility. The presence of the previously mentioned bacteria especially in the airways triggers a severe inflammatory response that irreversibly damages the lung [3]. So CF is a multi-organ, progressive disorder and its symptoms are diverse with varying severity [4].

The therapy of this chronic, progressive and multi-organ disorder is complex, lasts a lifetime and significantly influences the Quality of Life (QoL) of patients [5]. CF requires daily medical treatment, including physiotherapy and oral and inhaled respiratory medication to minimize deterioration of lung function, and dietary supplementation with pancreatic enzymes and vitamin supplements. The members of the available medicinal therapy include anti-infective medicinal products (e.g. antibiotics), anti-inflammatory drugs (especially non-steroidal agents), mucus alteration and airway surface liquid modulators (e.g. hypertonic saline), CTFR modulator (Kalydeco ${ }^{\circledR}$ ) and nutritional products (e.g. pancrelipase products) [6]. So, 
the daily management requires a complex, timeconsuming treatment regimen to address the multisystem effects of CF. In general, the long-term use of one or more pulmonary medications is recommended, which can include inhaled antibiotics (e.g. tobramycin, colistin, aztreonam) and oral azithromycin and nebulized mucolytic agents (e.g. dornase alfa, hypertonic saline) [7]. Several new potential therapy members are in the clinical trial phase of the development pipeline worldwide, such as gene and stem cell therapy, new smallmolecule CTFR modulators, new drugs in anti-infective and anti-inflammatory therapy [6], etc.

Patient characteristics: worldwide, the number of patients diagnosed with CF was 30,775 in the latest report [8]. Every year 900-100 new CF cases are discovered and diagnosed, the mean age of the newly diagnosed patients is about 4 years. The mean age of the patients registered with CF is 22.2 years. The predicted life expectancy (median survival age) is 44.4 years, but the median age at death is about 30 years according to the Cystic Fibrosis Foundation Patient Registry, Annual Data Report 2018 [8]. Several patients need transplantation. Lung transplantation is over-represented, more than 200 lung transplantations are carried out yearly for the CF reason, but kidney and liver transplantations can also be part of the treatment. The distribution among the races is the following: more than $90 \%$ (93.5\% in 2018) belongs to the white race and the remaining number is divided almost equally among the other races. The distribution of the disease among the genders is about $50-50 \%$ [8]. So CF disease is diagnosed mostly in childhood, in the preschool age, most of the patients are young adults or adolescents, and only a few percent of the patients reach the adult stage of life [9-11]. Therefore, how the patient can follow the complex therapy of CF strongly depends on their surroundings, family members, parents etc., their supportive attitude and help [12]. Co-morbidities and their therapy [7] are also an additional issue in this case. As the medical treatment for $\mathrm{CF}$ is demanding, support is essential. By adhering to the medical treatment, hospitalization can be decreased and the serious states can be avoided, which are crucial for QoL expectations in CF $[5,13]$.

\subsection{Adherence}

The World Health Organization (WHO) defines adherence as "the extent to which the persons' be- havior (including medication-taking) corresponds with agreed recommendations from a healthcare provider" [14]. or in a more detailed definition: "the extent to which a person's behavior - taking medication, following a diet, and/or executing lifestyle changes, corresponds with agreed recommendations from a health care provider" [14]. If the patient adheres (or holds) to the therapy, it means his/her active attendance and own responsibility in achieving improvement in his/her health status or quality of life while having a chronic disease. Previously, the commonly used term was "compliance" in relation to the patient's habit, but compliance describes a passive behavior.

Adherence to a long-term therapy in a chronic disease is a dynamic process which needs to be followed up. Family, community and patients' organizations are the key factors for having success in improving adherence, which is essential. Poor adherence to long-term therapies lead to poor health outcomes and increase health care costs, and the patients need to be supported. Adherence is simultaneously influenced by several factors and a multidisciplinary approach is needed to evaluate it [14].

The WHO defined 5 dimensions (groups) of the influencing factors of patient adherence [14]. The following groups influence the patient's adherence to a long-term therapy: (1) Social and economic factors, (2) Therapy-related factors, (3) Patient-related factors, (4) Condition-related factors and (5) Health system (health care system)-related factors.

\subsection{Quality by Design}

The Quality by Design (QbD) approach is a holistic, systemic, knowledge and Risk Assessment (RA) focused method used in the pharmaceutical industry [15] as its application is now a regulatory requirement for marketing authorization. The exact definition of $\mathrm{QbD}$ according to the International Council on Harmonisation (ICH) Quality Guidelines is as follows: "QbD is a systematic approach to development that begins with predefined objectives and emphasizes product and process understanding and process control, based on sound science and quality risk management" [16].

This risk-based model can be applied and adapted in each segment of the entire pharma sector, can be used in the pharmaceutical research and development area, and can also be extended 
[17]. The QbD method is a complex process and has several steps, which are defined and described in the ICH Q8(R2), Q9 and Q10 guidelines [16-19].

Besides its risk and knowledge focused feature, the first emphatic element of the $\mathrm{QbD}$ approach is the profound previous target product design. Compared to the classical Quality by Testing $(\mathrm{QbT})$ pharmaceutical model, the phase of this prudent theoretical product design is extremely expanded in the QbD-based development and it contains requirements, expectations, and needs not only from the industry and regulatory body but also form the clinical users (patients, doctors), which are in strong relationship with the patient's adherence in the future. Such patient's expectations and needs can be e.g. the changing of the dosage form, the potentials of another dosing regimen, the easy application of the product, or an alternative route of administration, the elimination of side effects or the elimination of unpleasant other effects or product characteristics.

\subsection{Aims}

The first aim of this study was the analysis of the needs and requirements in CF therapy and the implementation of these findings in the early development phase of pharmaceutical development in this area. The further aim was the application of the $\mathrm{QbD}$ guided risk-based approach in the medicinal product design in $\mathrm{CF}$ therapy according to the extended methodology of the QbD approach [17] in order to improve patient adherence and quality of life of patients who suffer from CF.

\section{Methods}

\subsection{Data collection, knowledge space development}

The screening, analysis and summarizing of the relevant literature in $\mathrm{CF}$ therapy management was made for the knowledge space development [20] and for the new therapeutic item design in CF. For literature screening the online scientific portals, like Elsevier, PubMed, ResearchGate and other literature database were used.

The relevant factors of $\mathrm{CF}$ therapy development were collected with special attention to patient expectations, adherence-related factors, therapy-specific elements and regulatory elements.

For the visualization of the collected items modern quality management tools, like the Ishikawa diagram [21], set formation, flowchart building and Pareto charts [22] were used. The Ishikawa diagram is a tool for evaluating the cause and effects relationship among the factors collected, and the Pareto diagram can help in the visualized ranking of the factors with critical effect on the aimed product, and to find those which have the highest criticality.

\subsection{Risk assessment}

The LeanQbD ${ }^{\circledR}$ software (QbD Works LLC, Fremont, CA, USA) was used for the Risk Assessment process in order to determine the factors with the highest risk. To carry out the RA properly, first those factors were selected which have a potential critical effect on therapy development or management in CF. The Ishikawa diagram was used for the selection of the items. The selected critical factors were grouped into two groups: Group 1: factors which have an effect on QoL in CF (= Critical Quality Attributes, CQAs), Group 2: factors which have an effect on the therapy, or the therapeutic process of $\mathrm{CF}$ (= Critical Process Parameters, CPPs). After the above-mentioned item selection and grouping, an interdependence rating was made among the elements step by step, pairwise. A three-level scale was used to describe the relation between the parameters, so the interaction was described as "high" $(\mathrm{H})$, "medium" (M) or "low" (L). This was followed by an uncertainty rating step, in which CPPs were estimated and categorized by the same three-grade scale. As a result of the RA, Pareto charts were generated by the software, presenting the numeric data and the ranking of the CQAs and CPPs according to their criticality.

\section{Results}

This study aimed to perform a systemic evaluation of the patient and regulatory needs in $\mathrm{CF}$ therapy development. So first, in order to fulfill all the elements of knowledge management and knowledge space development, the adherence factors were visualized according to the WHO dimensions (Figure 1).

After this global view of adherence, the disease and therapy-specific sub-dimensions in CF were collected, systemized and visualized by setting up an Ishikawa diagram, presenting the cause and effects relationships (Figure 2).

Set formation was the additional tool for visualizing the knowledge and the influencing factors in 


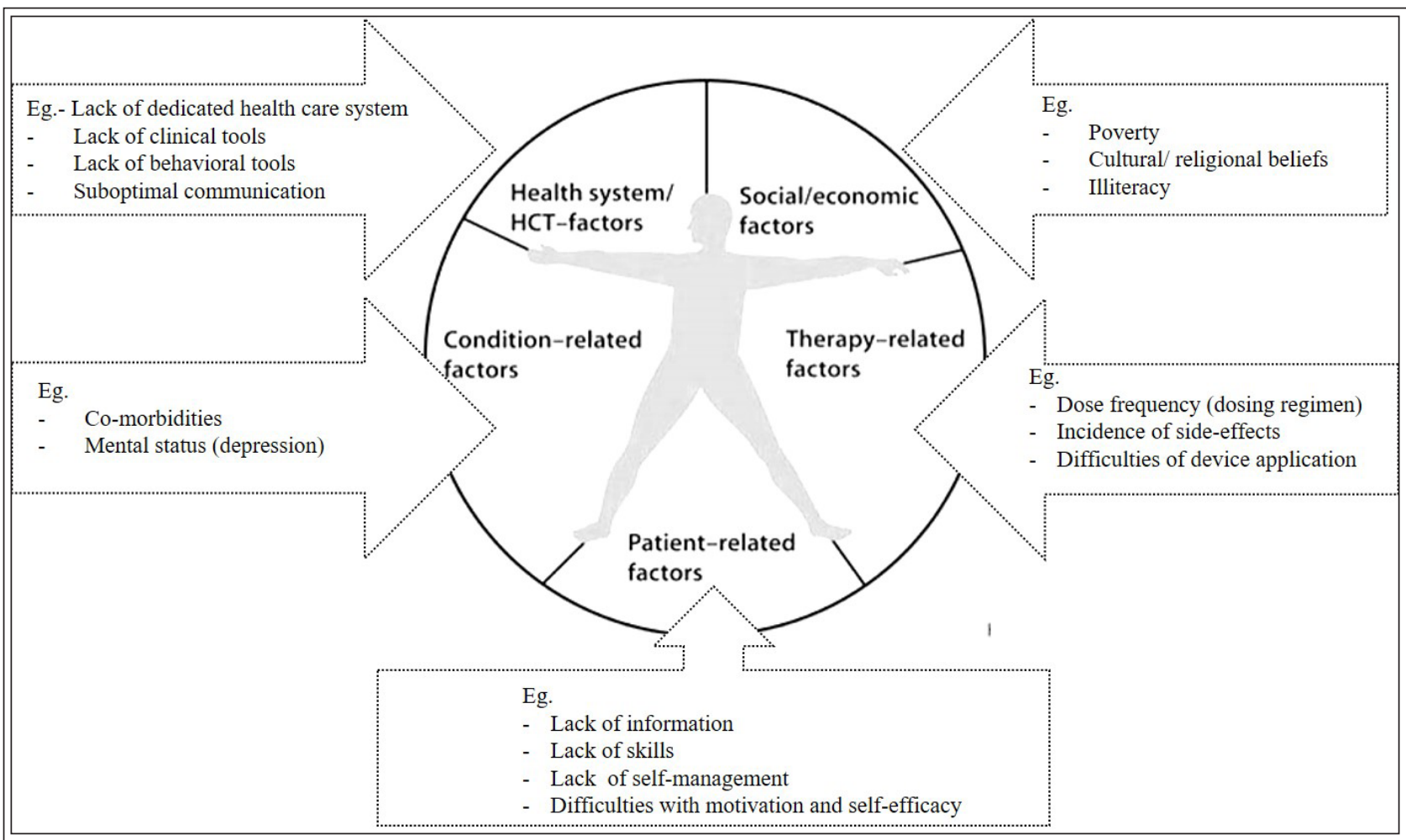

Figure 1 The 5 dimensions and their potential elements of adherence by the WHO

CF treatment or therapy development and for analyzing the interactions. The factors of patient adherence presented previously formed one of the sets, complemented with two other groups: the relevant economic and regulatory standpoints.

The most important elements from the regulatory viewpoint in a new CF-medicine or therapy development are: $\mathrm{CF}$ belongs to the rare or orphan diseases, which means a low number of cases, a low number of patients, so this is a narrow market area. The low number of patients also affects the clinical trials, there could be difficulties in selecting patients who can be involved in the study, and it may lead to problems in keeping the anonymity of the patients [23]. The planning of the clinical studies also needs special considerations: there is no possibility of placebo control and randomization during the studies, and questions arise regarding the end-point definition. The low case-number in the clinical trial phase necessitates other solutions to obtain the results about the safety and efficacy of the investigated product ( $=$ the potential new medicinal product), which are required for the documentation of the authorization. In practice, this means that the importance and the rate of the statistical analysis, modeling and simulation are increased, and their use is essential. This special situation generated by the limited source of the clinical data needs special considerations, effective communication and co-operation during the whole process of the orphan medicine development [23], from drug design through dosage formulation to the clinical phases, and needs the involvement of the regulatory/authorization body as well.

The economic standpoints are in relation with the costs: costs of the development, cost of the new medicinal product, cost of the therapy. The therapeutic cost (TC) of CF is very high and diverse, the averaged costs are presented in Figure 3 as well. The total TC for the whole life can reach almost 2 million Euros [24], but the range of the total TC is very wide. When several hospitalizations are needed during the lifetime and transplantation is also required, this total TC can reach the upper limit of this range [24]. The TC/year and the health care costs/year are also shown in the figure.

The total cost includes direct costs, indirect costs and intangible costs [25]. Direct costs include the costs of medication and obtaining care (e.g. costs of visits to physicians and other health care providers, hospitalizations, medications and other treatments, assistive devices and diagnostic tests, as well as the costs of transportation, etc.). Indirect costs are associated with patient or carer productivity losses. Intangible costs represent the costs of reductions in quality of life due to the ill- 


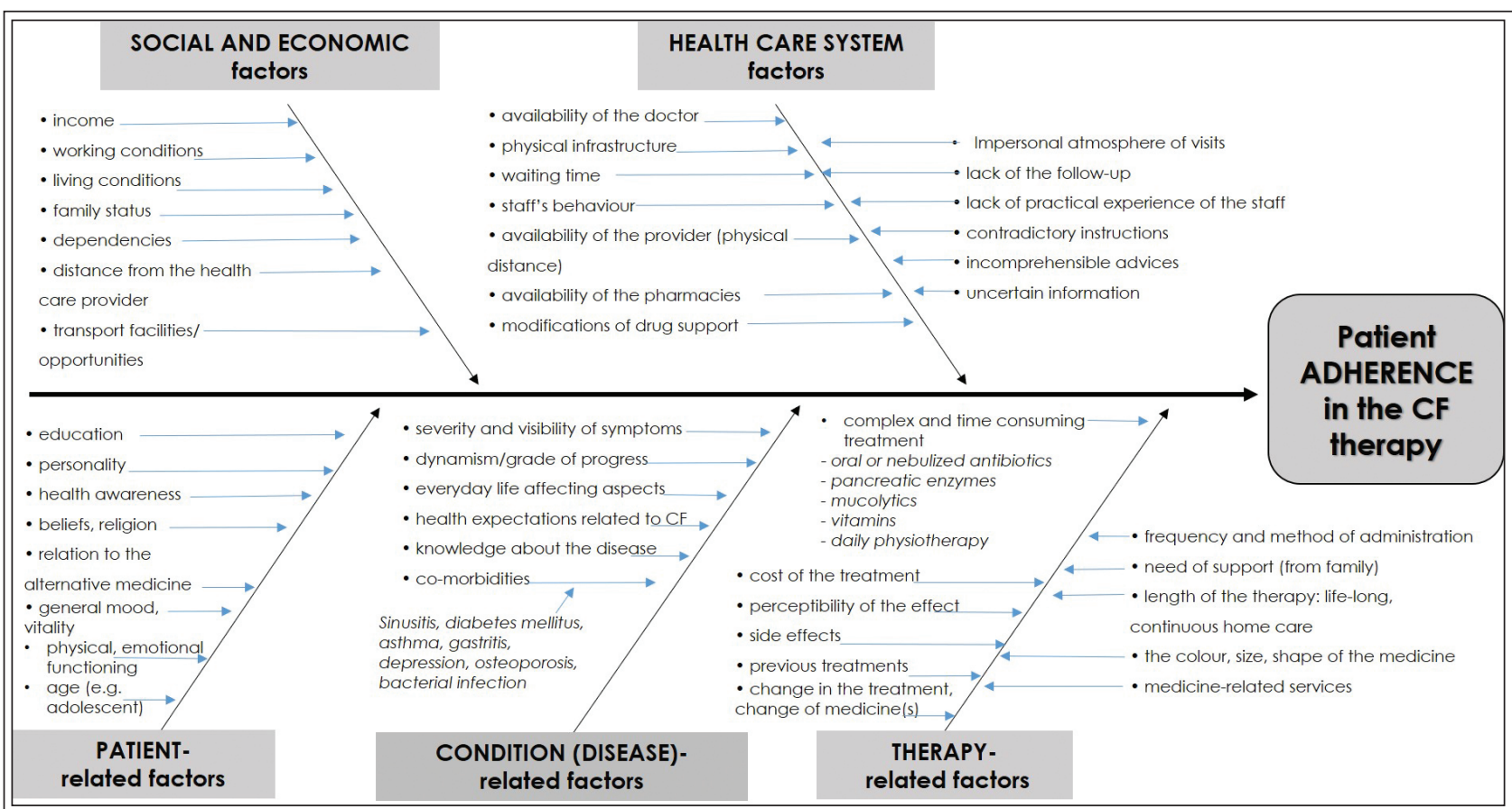

Figure 2 Sub-dimensions of the WHO groups of patient adherence in CF disease

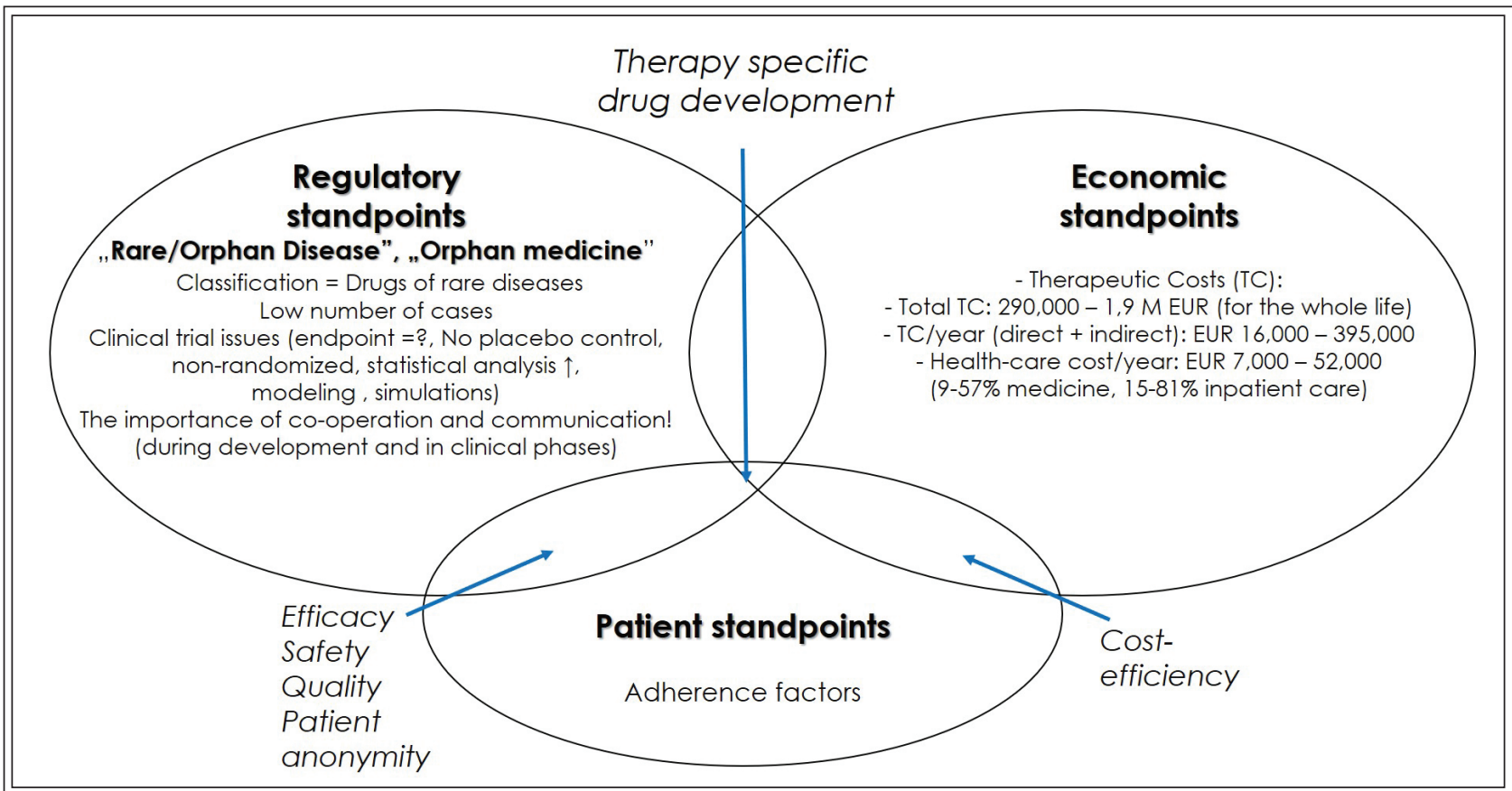

Figure 3 Regulatory and economic standpoints in CF therapy development

ness, but as these are difficult to quantify economically, intangible costs are rarely estimated.

The cross sections of the sets in Figure 3 include the most critical elements of the CF-specific therapy development concerning the efficacy, safety and quality of the newly developed formula, patient security aspects, like anonymity and clinical study competence, and cost efficiency [11, $23,24]$.
The literature evaluation and data collection which are presented in Figure 2 and Figure 3 helped in the selection of the critical factors of QoL and therapy of CF for the risk-based evaluation of the development potentials. So, the factors were classified into two groups for the evaluation. The first group contains those factors which could have a critical influence on QoL in CF, these selected CQAs are the following: the age of the pa- 


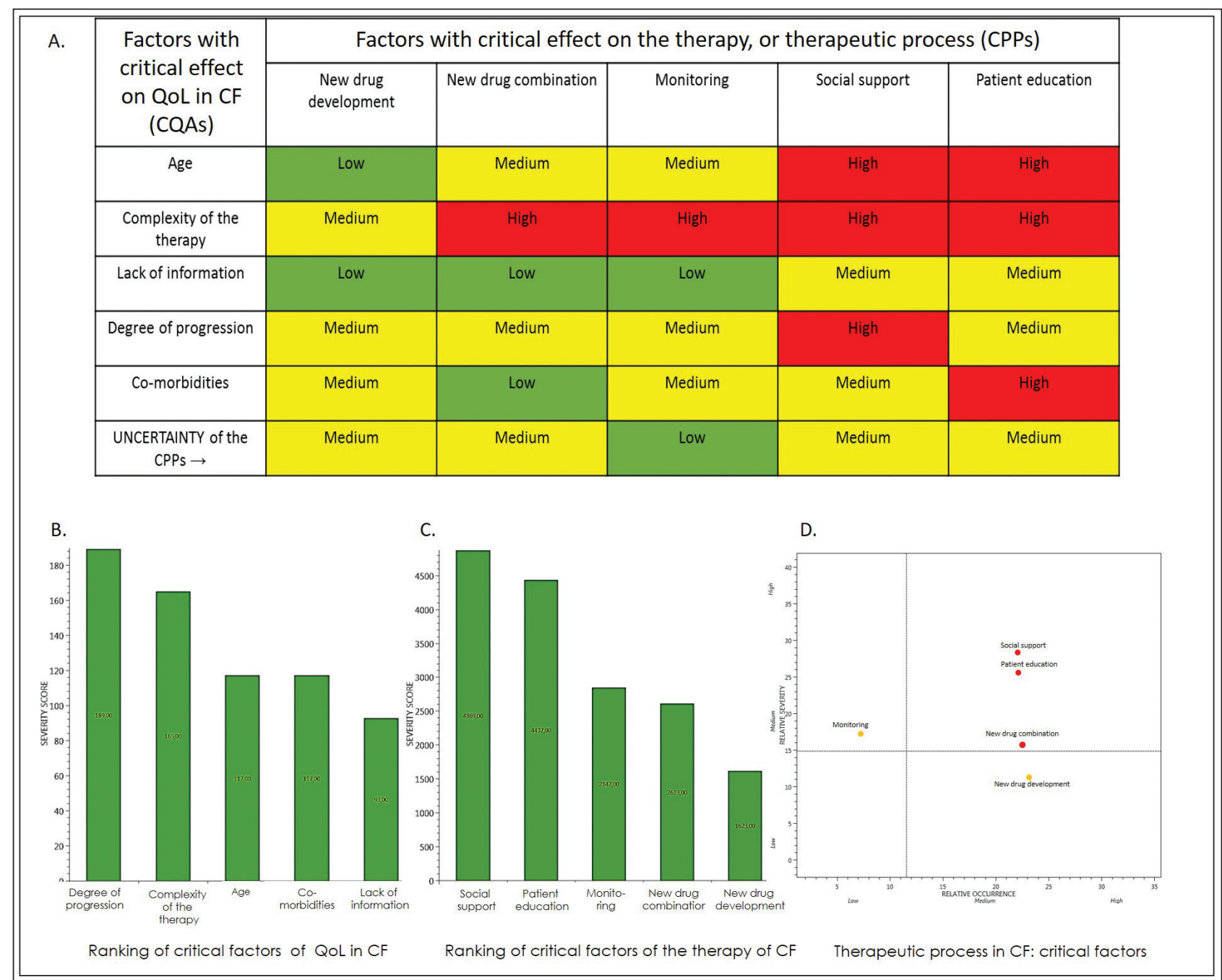

Figure 4 Risk estimation matrix $(A)$ and the ranking of the critical parameters in $C F$ therapy development $(B, C, D)$

tient who suffers from $\mathrm{CF}$, their co-morbidities, the degree of progression, the complexity of the therapy and the lack of information (about the therapy, expectations, disease, etc.). Those elements were categorized into the second group which could have a critical influence on the therapy (as process) and therapeutic success of CF. This group of the CPPs contained the following: new drug product development (new active agent, new administration method, new dosage form, etc.), new drug combination development, patient education, social support, and the monitoring of the therapy.

The grouping of the factors was essential to perform the RA properly, which was made with the help of the RA software. The first step was an interdependence rating among the factors in pairs, where their effect on each other was estimated (high, medium or low), which was followed by an occurrence rating, using the same three-grade scale in estimation. This is presented in the risk estimation matrix (REM) in Figure 4-A.

As the result of the risk estimation, the software calculated the severity scores of each factor and generated Pareto charts for the visualization of the calculated theoretical ranking of the examined critical factors. Figure 4 presents their ranking according to their criticality on the patients' quality of life in CF (Figure 4-B) and on the therapy of CF (Figure 4-C). A relative severity and relative occurrence diagram were also generated by the software (Figure 4-D), where the red points show the factors with the highest critical effect on the therapy. The results presented in Figure 4 show the following: with regard to the patient's QoL in CF, the degree of progression of the disease has the highest criticality, followed by the complexity of the therapy (e.g. dosing regimen), a medium critical effect was found in relation to the patient's age and co-morbidities, while the lack of the informa- 


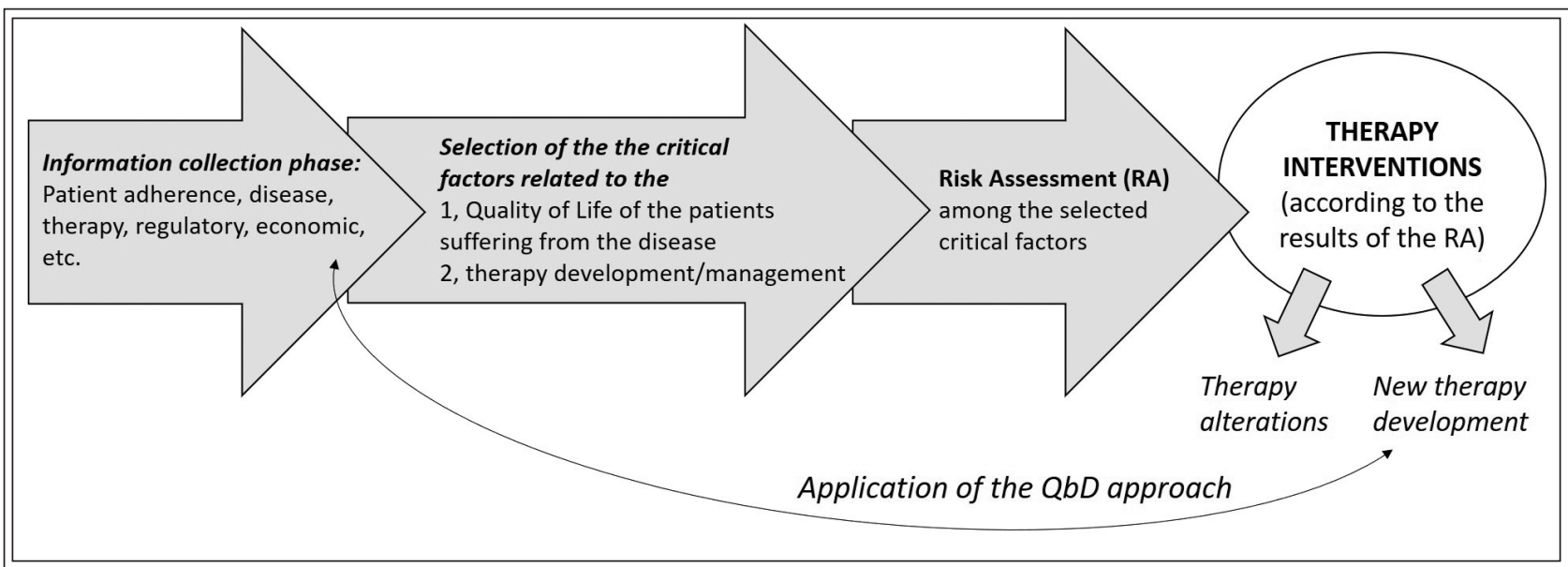

Figure 5 Flowchart of a risk assessment-based therapy intervention design

tion of the patients was found to have the lowest critical effect. So the greatest attention needs to be focused on decreasing progression and on simplifying the complexity of the therapy in order to increase the perception of the QoL and adherence among the patients.

In the case of the therapy-related critical factors in CF, the social support factor was found to have the highest critical effect. Social support (e.g. family) or the lack of it has a great effect on successful therapy. It is followed by patient education, which also has a high critical effect. Monitoring and the new drug combination have a medium critical effect, they are placed in the middle of the ranking, and the lowest criticality was found for the development of a new drug product.

\section{Discussion}

the risk-based evaluation presented in this study and the RA results showed that the recommended intervention points in $\mathrm{CF}$ therapy management are the following: increasing social support, increasing the level of patient education and improving the potentials of therapy monitoring. The degree of adherence in $\mathrm{CF}$ depends mainly on the degree of progression and the complexity of the therapy. The steps of the evaluation method used are visualized and shown in Figure 5. This model is based on the QbD method, which is generally used in the pharmaceutical industry, but its theoretical background, namely the systemic, knowledge and riskbased design of tasks can be applied to risk-based therapy management as well (Figure 5).

The interventions identified by the risk-based evaluation of therapy development or management which can improve the patients' quality of life may be the alternative of costly and time-consuming new drug developments.

\section{Conclusion}

In this study the application of a QbD and riskbased therapy-specific drug development design was presented in CF therapy. To evaluate the aspects which affect the QoL and therapy success (adherence) in this disease, the patient's, regulatory and economic standpoints of therapy development were collected. The overlap of these aspects has great importance with regard to meeting the requirements of efficacy, safety and quality standards as well as the cost-efficiency considerations. According to the presented results, if therapy intervention is needed in $\mathrm{CF}$, one should first examine the patient's social support state and find possibilities to improve it. Then patient education should be improved, followed by the upgrading of therapy monitoring or introducing a new one. Only after these should the potential need for new pharmaceutical development, such as a new drug combination or new medicine development, be considered. Sometimes such interventions (in the lifestyles, habits, dosing regimen modifications, education, patient education, etc.) may be the alternatives of a time-consuming and costly medicinal research and development. The presented model can be used for the evaluation of therapy potentials in several other diseases as well.

\section{Acknowledgment}

This study was supported by the EFOP 3.6.2-162017-0006. Authors express their gratitude for it. 


\section{List of Abbreviations}

CF: Cystic fibrosis

CPPs: Critical Process Parameters

CQAs: Critical Quality Attributes

CTFR: cystic fibrosis transmembrane conductance regulator

ICH: International Council on Harmonisation

QbD: Quality by Design

QoL: Quality of Life

RA: Risk Assessment

WHO: World Health Organization

\section{References}

1. K De Boeck, M Wilschanski, C Castellani, C Taylor, $\mathrm{H}$ Cuppens, J Dodge MS. Cystic fibrosis : terminology and diagnostic algorithms- Occasional Review. Thorax. 2006;61:627-635. https://doi.org/10.1136/ thx.2005.043539

2. Ren CL, Morgan RL, Oermann C, et al. Cystic Fibrosis Foundation Pulmonary Guidelines Use of Cystic Fibrosis Transmembrane Conductance Regulator Modulator Therapy in Patients with Cystic Fibrosis. Clin Pract Guidel. 2018;15(3):271-280. https://doi. org/10.1513/AnnalsATS.201707-539OT

3. Galietta LJ V. Managing the Underlying Cause of Cystic Fibrosis: A Future Role for Potentiators and Correctors. Pediatr Drugs. 2013;(15):393-402. https://doi. org/10.1007/s40272-013-0035-3

4. EMA, CHMP. Guideline on the clinical development of medicinal products for the treatment of cystic fibrosis. Www.emea.europa.eu. 2009;(October).

5. Smyth AR, Bell SC, Bojcin S, et al. European cystic fibrosis society standards of care: Best practice guidelines. J Cyst Fibros. 2014;13(S1):S23-S42. https://doi. org/10.1016/j.jcf.2014.03.010

6. Hoffman LR, Ramsey BW. Cystic Fibrosis Therapeutics, The Road Ahead. Chest. 2013;(January):207-213. https://doi.org/10.1378/chest.12-1639 7.

7. Quittner AL, Zhang J, Marynchenko M, Chopra PA. Pulmonary Medication Adherence and Health-care Use in Cystic Fibrosis. Chest. 2014;146(1):142-151. https://doi.org/10.1378/chest.13-1926

8. Cystic Fibrosis Foundation. Patient Registry Annual Data Report. Lung. 2015. http://www.cff.org/UploadedFiles/research/ClinicalResearch/Patient-RegistryReport-2009.pdf.

9. Eakin MN, Riekert KA. The impact of medication adherence on lung health outcomes in cystic fibrosis. Curr Opin Pulm Med. 2017;19(6):687-691. https://doi. org/10.1097/MCP.0b013e3283659f45

10. Kettler LJ, Sawyer SM, Winefield HR, Greville HW. Determinants of adherence in adults with cystic fibrosis- occasional review. Thorax. 2002;57(October 2001):459-464. https://doi.org/10.1136/tho$\operatorname{rax} .57 .5 .459$

11. King CS, Brown AW, Aryal S, Ahmad K. Critical Care of the Adult Patient With Cystic Fibrosis.
Chest. 2019;155(1):202-2014. https://doi.org/10.1016/j. chest.2018.07.025

12. Bregnballe V, Schiøtz PO, Boisen KA, Pressler T, Thastum M. Barriers to adherence in adolescents and young adults with cystic fibrosis: A questionnaire study in young patients and their parents. Patient Prefer Adherence. 2011;5:507-515. https://doi.org/10.2147/PPA.S25308

13. De Jong W, Kaptein AA, Van Der Schans CP, et al. Quality of life in patients with cystic fibrosis. Pediatr Pulmonol. 1997;23(2):95-100. doi:10.1002/(SICI)10990496(199702)23:2<95::AID-PPUL4>3.0.CO;2-N https://doi.org/10.1002/(SICI)1099-0496(199702) 23:2<95::AID-PPUL4>3.0.CO;2-N

14. WHO. Adherence to Long-Term Therapies, Evidence for Action. Switzerland; 2003. https://doi.org/10.4028/ www.scientific.net/AMM.321-324.1779

15. Yu LX. Pharmaceutical quality by design: Product and process development, understanding, and control. Pharm Res. 2008;25(4):781-791. https://doi. org/10.1007/s11095-007-9511-1

16. ICH. Pharmaceutical Development Q8. ICH Harmon Tripart Guidel. 2009;8(August):1-28.

17. Csóka I, Pallagi E, Paál TL. Extension of quality-by-design concept to the early development phase of pharmaceutical R\&D processes. Drug Discov Today. 2018. https://doi.org/10.1016/j.drudis.2018.03.012

18. ICH. Quality Risk Management Q9. ICH Harmon Tripart Guidel. 2005:1-23. https://doi.org/10.1007/s11095007-9511-1

19. ICH. ICH Q10 Pharmaceutical Quality Systems. EPTThe Electron Newsl Pharm Tech Jun. 2009;(May):21. https://doi.org/10.1007/978-3-319-15814-3

20. Pallagi E, Karimi K, Ambrus R, Szabó-Révész P, Csóka I. New aspects of developing a dry powder inhalation formulation applying the quality-by-design approach. Int J Pharm. 2016;511(1). https://doi.org/10.1016/j.ijpharm.2016.07.003

21. Tague NR. Fishbone Diagram (Ishikawa) - Cause \& Effect Diagram. Qual Toolbox. 2005:247-249. http://asq. org/learn-about-quality/cause-analysis-tools/overview/fishbone.html.

22. Powell T, Sammut-Bonnic T. Pareto Analysis. In: Cooper. CL, ed. Wiley Encyclopedia of Management. John Wiley \& Sons, Ltd; 2014. https://doi. org/10.1002/9781118785317.weom120202

23. Mulberg AE, Bucci-rechtweg C, Giuliano J, et al. Regulatory strategies for rare diseases under current global regulatory statutes: a discussion with stakeholders. Orphanet J Rare Dis. 2019;5:14-36. https://doi. org/10.1186/s13023-019-1017-5

24. Angelis A, Tordrup D, Kanavos P. Socio-economic burden of rare diseases: A systematic review of cost of illness evidence. Health Policy (New York). 2015;119(7):964-979. https://doi.org/10.1016/j.healthpol.2014.12.016

25. Jo C. Cost-of-illness studies: concepts, scopes, and methods. Clin Mol Hepatol. 2014;20(4):327-337. https://doi.org/10.3350/cmh.2014.20.4.327 